\title{
The Lie Algebra of Local Killing Fields
}

\author{
Richard Atkins ${ }^{*}$
}

Department of Mathematics, Trinity Western University, 7600 Glover Road, Langley, BC, V2Y 1Y1, Canada

\begin{abstract}
We present an algebraic procedure that finds the Lie algebra of the local Killing fields of a smooth metric. In particular, we determine the number of independent local Killing fields about a given point on the manifold. As an application, we provide a local classification of the types of surfaces that admit the various possible Lie algebras of local Killing fields, in terms of the Gaussian curvature.
\end{abstract}

Keywords: Lie algebra, local Killing field, derived flag, curvature, locally symmetric space.

\section{INTRODUCTION}

Killing fields describe the infinitesimal isometries of a metric and as such play a significant role in differential geometry and general relativity. In this paper we present an algebraic method that finds the Lie algebra of the local Killing fields of a smooth metric $g$. In particular, we determine the number of independent local Killing fields of $g$ about any given point. In the section following, we identify the local Killing fields of a metric with local parallel sections of an associated vector bundle $W$, endowed with a connection $\nabla$. An examination of the form of the curvature of $\nabla$ leads to a characterization of spaces of constant curvature by means of a system of linear equations. In Section 3 we investigate the Lie algebra structure of Killing fields. Section 4 includes an overview of the procedure developed in [1]. Therein the bundle generated by the local parallel sections of $W$ is found by calculating a derived flag of subsets of $W$. The number of independent Killing fields of $g$ about a point $x \in M$ is then equal to the dimension of the fibre $\widetilde{W}_{x}$ over $x$ of the terminal subset of the derived flag. Associated to $\widetilde{W}_{x}$ is a Lie algebra canonically isomorphic to the Lie algebra $K_{x}$ of local Killing fields about $x$. The method is illustrated by providing a short proof of a classical theorem that gives a necessary condition for a space to be locally symmetric, expressed by the vanishing of a set of quadratic homogeneous polynomials in the curvature. Section 5 considers the derived flag for surfaces. We obtain a complete classification of the local Riemannian metrics corresponding to the various possible kinds of Lie algebra $K_{x}$. Furthermore, we show that if a regular surface with non-constant curvature possesses a Killing field then the integral curves of the curvature vector field are geodesic paths.

*Address correspondence to this author at the Department of Mathematics, Trinity Western University, 7600 Glover Road, Langley, BC, V2Y 1Y1 Canada; Tel: 1-604-888-7511; Fax: 1-604-513-2018; E-mail: richard.atkins@twu.ca

\section{KILLING FIELDS AND CONSTANT CURVATURE}

We associate to Killing fields parallel sections of a suitable vector bundle in the manner put forward by Kostant [2]. The utility of such a framework is two-fold: first, it permits us to apply algebraic techniques adapted to finding the subbundle generated by local parallel sections. Second, it enables a purely algebraic description of the Lie bracket of two Killing fields, avoiding the explicit appearance of derivatives.

Let $g$ be a metric on a differentiable manifold $M$ of dimension $n ; g$ is assumed to be pseudo-Riemannian of signature $(p, q)$ unless otherwise stated. $K$ is a Killing field of $g$ if and only if,

$$
K_{a ; b}+K_{b ; a}=0
$$

where the semi-colon indicates covariant differentiation with respect to the Levi-Civita connection of $g$. It is straightforward to verify that,

$$
K_{a ; b c}=R_{a b c}{ }^{d} K_{d}
$$

for Killing fields $K$, where $R_{a b c}{ }^{d}$ is the Riemann curvature tensor of $g$, defined according to,

$$
A_{c ; b a}-A_{c ; a b}=R_{a b c}{ }^{d} A_{d}
$$

The summation convention shall be used throughout.

Let $W$ be the Whitney sum $W:=T^{*} M \oplus \Lambda^{2} T^{*} M$. A local section of $W$ has the form $X=K+L$, where $K=K_{a} d x^{a}$ is a local section of $T^{*} M$ and $L=L_{a b} d x^{a} \wedge d x^{b}$ is a local section of $\Lambda^{2} T^{*} M$. Define a connection $\nabla$ on $W$ by,

$\nabla_{i} X=\left(K_{a ; i}-L_{a i}\right) d x^{a}+\left(L_{a b ; i}-R_{a b i}^{c} K_{c}\right) d x^{a} \wedge d x^{b}$

For an open subset $U \subseteq M$, let $K_{U}$ denote the local Killing fields $K: U \rightarrow T^{*} M$ and let $P_{U}$ denote the local parallel sections $X: U \rightarrow W$; the subscript $U$ shall be omitted when $U=M$. Define the map $\phi_{U}: K_{U} \rightarrow P_{U}$ by, 
$\phi_{U}\left(K_{a}\right):=K_{a}+K_{a ; b}$

It is clear that the image of $\phi_{U}$ does, in fact, lie in $P_{U}$. The inverse $\psi_{U}: P_{U} \rightarrow K_{U}$ of $\phi_{U}$ is the projection of $W$ onto $T^{*} M: \psi_{U}\left(K_{a}+L_{a b}\right):=K_{a}$. This establishes a vector space isomorphism,

$K_{U} \leftrightarrow P_{U}$

Consider a vector space $V$ with a non-degenerate, symmetric bilinear form $h$. Let $B=B_{a b c d}$ be a covariant 4 tensor on $V$ satisfying the following relations common to a Riemann curvature tensor:

$B_{a b c d}=B_{c d a b}=-B_{b a c d}=-B_{a b d c}$

and let $T=T_{a b \ldots}$ be an $n$-tensor on $V$ with $n \geq 2$. The derivation $B \star T$ is the $(n+2)$-tensor defined by,

$B \star T_{a b c d \cdots}:=B_{s b c d} T_{a \cdots}^{s}+B_{a s c d} T_{b \cdots}^{s}+B_{a b s d} T_{c \cdots}^{s}+B_{a b c s} T_{d \cdots}^{s}$

Indices are raised by $h$.

Lemma 1. If $V$ is 2-dimensional then $B \star L=0$ for all $L \in \Lambda^{2} V^{*}$.

\section{Proof:}

It shall be convenient to work in an orthonormal basis of $V$ in which $h=\operatorname{diag}\left(\eta_{1}, \eta_{2}\right)$, where $\eta_{i}= \pm 1$. Then $L_{j}^{i}=\eta_{i} L_{i j}$. Owing to the symmetries (6), there are effectively two cases to consider.

(i) $a=b=1$ case:

$$
B \star L_{a b c d}=\eta_{2}\left(B_{21 c d}+B_{12 c d}\right) L_{21}=0
$$

(ii) $a=c=1, b=d=2$ case:

$B \star L_{a b c d}=\eta_{2} B_{2212} L_{21}+\eta_{1} B_{1112} L_{12}+\eta_{2} B_{1222} L_{21}+\eta_{1} B_{1211} L_{12}=0$

q.e.d.

Applying the Bianchi identities, the curvature $F(i, j):=\nabla_{[i} \nabla_{j]}$ of $\nabla$ takes the form,

$F(i, j)(X)=\left(R_{i j k l ; s} K^{s}+R \star L_{i j k l}\right) d x^{k} \wedge d x^{l}$

where $X=K_{a} d x^{a}+L_{a b} d x^{a} \wedge d x^{b} \quad$ [3]. In the sequel, it shall be convenient to view the curvature $F$ as a map $F: W \rightarrow \Lambda^{2} T^{*} M \otimes W$ given by $w \mapsto F(),(w) . F$ is composed of two pieces: a $K$-part and an $L$-part. The $K$-part provides a description of locally symmetric spaces: $g$ is locally symmetric if and only if $T^{*} M \subseteq \operatorname{ker} F$. The $L$-part, on the other hand, provides a characterization of metrics of constant sectional curvature by means of a system of homogeneous linear equations.

Proposition 2. Let $g$ be Riemannian and $n \geq 3$. Then $M$ is a space of constant curvature if and only if,

$$
R \star L=0
$$

for all $L \in \Lambda^{2} T^{*} M$
Expressed in terms of indices, $M$ has constant curvature (for $n \geq 3$ ) if and only if for all $L \in \Lambda^{2} T^{*} M$,

$R_{s j k l} L_{i}^{s}+R_{i s k l} L_{j}^{s}+R_{i j s l} L_{k}^{s}+R_{i j k s} L_{l}^{s}=0$

It is evident from the lemma that the proposition does not hold for $n=2$.

Proof:

$\Rightarrow \quad$ If $\quad g$ has constant curvature then $R_{i j k l}=\kappa_{0}\left(\delta_{i l} \delta_{j k}-\delta_{i k} \delta_{j l}\right) \quad$ with respect to an orthonormal frame, where $\kappa_{0}$ is a constant. Substitution of this expression into the left hand side of (9) gives zero for all skew-symmetric $L=L_{a b}$.

$\Leftarrow$ Suppose that (9) holds for all $L \in \Lambda^{2} T^{*} M$. We shall work in an orthonormal frame $X_{1}, \ldots, X_{n}$ for $g$; this will allow us to deal with lowered indices throughout: $L_{j}^{i}=L_{i j}$. Let $i=k, j$ and $l$ be three distinct indices in (9). This gives,

$R_{s j l} L_{s i}+R_{i s i l} L_{s j}+R_{i j s l} L_{s i}+R_{i j i s} L_{s l}=0$

Put $L_{r s}:=\delta_{r l} \delta_{s j}-\delta_{r j} \delta_{s l}$ into (10) to obtain $R_{i j i j}=R_{i l i l}$. It follows that for any two pairs of distinct indices $i \neq j$ and $a \neq b, R_{i j i j}=R_{a b a b}$. Thus,

$R_{i j i j}=\kappa(x) \quad$ for $i \neq j$

where $\kappa$ is some function on $M$.

Next, let $i=k$ and $j=l$ be two distinct indices in (9). This gives:

$R_{i j i s} L_{s j}+R_{i j s j} L_{s i}=0$

Let $m$ be any index distinct from $i$ and $j$ and put $L_{r s}:=\delta_{r m} \delta_{s j}-\delta_{r j} \delta_{s m}$ into (12). We obtain,

$R_{i j i m}=0$ for $i, j$ and $m$ distinct

Consider a pair $Y_{1}, Y_{2}$ of orthonormal vectors in $T_{x} M$. If $X_{1}, X_{2}$ span the same plane as $Y_{1}, Y_{2}$ at $x$ then $R\left(Y_{1}, Y_{2}, Y_{1}, Y_{2}\right)=\kappa(x)$, by (11). If $Y_{1}, Y_{2}$ span a plane orthogonal to $X_{1}, X_{2}$ then we may as well suppose $X_{3}=Y_{1}$ and $X_{4}=Y_{2}$, whence $R\left(Y_{1}, Y_{2}, Y_{1}, Y_{2}\right)=\kappa(x)$, from (11) again.

The last possibility is that $Y_{1}, Y_{2}$ and $X_{1}, X_{2}$ span planes that intersect through a line, which for the purpose of calculating sectional curvature we may take to be generated by $X_{1}=Y_{1}$, by means of appropriate rotations of the pairs $X_{1}, X_{2}$ and $Y_{1}, Y_{2}$ within the respective planes they span. We may suppose, furthermore, that $X_{3}$ is the normalized component of $Y_{2}$ orthogonal to $X_{2}$; thus,

$$
Y_{2}=a X_{2}+b X_{3} \text {, }
$$

where $a^{2}+b^{2}=1$. From (11) and (13) this gives, 
$R\left(Y_{1}, Y_{2}, Y_{1}, Y_{2}\right)=R\left(X_{1}, a X_{2}+b X_{3}, X_{1}, a X_{2}+b X_{3}\right)$

$=a^{2} R_{1212}+b^{2} R_{1313}$

$=\kappa(x)$

Therefore $g$ has constant curvature at each point $x \in M$. By Schur's Theorem, $g$ has constant curvature. q.e.d.

\section{THE LIE ALGEBRA STRUCTURE OF $K_{U}$}

Let $V$ be an $n$-dimensional vector space equipped with a non-degenerate, symmetric bilinear form $h$, of signature $(p, q)$, and let $B=B_{a b c d}$ be a covariant 4-tensor on $V$ satisfying the usual algebraic relations of a Riemann curvature:

$B_{a b c d}=-B_{b a c d}$

$B_{a b c d}=-B_{a b d c}$

$B_{a b c d}+B_{a c d b}+B_{a d b c}=0$, and afortiori

$B_{a b c d}=B_{c d a b}$

By virtue of (14) and (15) we may define a skewsymmetric, bilinear bracket operation on $V^{*} \oplus \Lambda^{2} V^{*}$ by,

$\left[K_{a}+L_{a b}, K_{a}^{\prime}+L_{a b}^{\prime}\right]$ :

$=L_{a b}^{\prime} K^{b}-L_{a b} K^{\prime b}+L_{a}^{\prime c} L_{c b}-L_{a}^{c} L_{c b}^{\prime}+B_{a b c d} K^{c} K^{\prime d}$

where indices are raised and lowered with $h$. If a subspace $W$ of $V^{*} \oplus \Lambda^{2} V^{*}$ is closed with respect to the bracket and satisfies the Jacobi identity then we denote the associated Lie algebra by $A(W, B, h)$.

Lemma 3. Let $W$ be a subspace of $V^{*} \oplus \Lambda^{2} V^{*}$, closed with respect to the bracket operation. The Jacobi identity holds on $W$ if and only if for all $X=K+L, X^{\prime}=K^{\prime}+L^{\prime}$ and $X^{\prime \prime}=K^{\prime \prime}+L^{\prime \prime} \quad$ in $\quad W$, where $K, K^{\prime}, K^{\prime \prime} \in V^{*} \quad$ and $L, L^{\prime}, L^{\prime \prime} \in \Lambda^{2} V^{*}$,

$B \star L_{a b c d} K^{\prime c} K^{\prime \prime d}+B \star L_{a b c d}^{\prime} K^{\prime \prime c} K^{d}+B \star L_{a b c d}^{\prime \prime} K^{c} K^{\prime d}=0$

Proof:

Let $K, K^{\prime}, K^{\prime \prime} \in V^{*}$ and $L, L^{\prime}, L^{\prime \prime} \in \Lambda^{2} V^{*}$. There are four cases to consider.

(i) $K-K^{\prime}-K^{\prime \prime}$ case. We have,

$$
\left[K,\left[K^{\prime}, K^{\prime \prime}\right]\right]=\left[K, B_{a b c d} K^{\prime c} K^{\prime \prime d}\right]=B_{a b c d} K^{b} K^{\prime c} K^{\prime \prime d}
$$

Therefore,

$$
\begin{aligned}
& {\left[K,\left[K^{\prime}, K^{\prime \prime}\right]\right]+\left[K^{\prime},\left[K^{\prime \prime}, K\right]\right]+\left[K^{\prime \prime},\left[K, K^{\prime}\right]\right]} \\
& =\left(B_{a b c d}+B_{a c d b}+B_{a d b c}\right) K^{b} K^{\prime c} K^{\prime \prime d} \\
& =0
\end{aligned}
$$

by equation (16).

(ii) $K-K^{\prime}-L$ case. First,

$$
\left[K,\left[K^{\prime}, L\right]\right]=\left[K, L_{a b} K^{\prime b}\right]=B_{a b c d} K^{c} L_{s}^{d} K^{\prime s}
$$

Also,

$$
\begin{aligned}
{\left[L,\left[K, K^{\prime}\right]\right]=} & {\left[L, B_{a b c d} K^{c} K^{\prime d}\right] } \\
& =B_{a s c d} K^{c} K^{\prime d} L_{b}^{s}-L_{a}^{s} B_{s b c d} K^{c} K^{\prime d}
\end{aligned}
$$

Combining these with (15) and the fact that $L=L_{a b}$ is skew-symmetric, we obtain,

$$
\left[K,\left[K^{\prime}, L\right]\right]+\left[K^{\prime},[L, K]\right]+\left[L,\left[K, K^{\prime}\right]\right]=B \star L_{a b c d} K^{c} K^{\prime d}
$$

$$
\begin{aligned}
& K-L-L^{\prime} \text { case. Observe that, } \\
& {\left[K,\left[L, L^{\prime}\right]\right]=\left[K, L^{\prime} L-L L^{\prime}\right]=L^{\prime} L K-L L^{\prime} K}
\end{aligned}
$$

and,

$$
\left[L,\left[L^{\prime}, K\right]\right]=-\left[L, L^{\prime} K\right]=L L^{\prime} K
$$

Using these equations gives,

$\left[K,\left[L, L^{\prime}\right]\right]+\left[L,\left[L^{\prime}, K\right]\right]+\left[L^{\prime},[K, L]\right]=0$

(iv) $L-L^{\prime}-L^{\prime \prime}$ case. It is elementary to verify that,

$$
\left[L,\left[L^{\prime}, L^{\prime \prime}\right]\right]+\left[L^{\prime},\left[L^{\prime \prime}, L\right]\right]+\left[L^{\prime \prime},\left[L, L^{\prime}\right]\right]=0
$$

After applying (19)-(22),

$$
\left[X,\left[X^{\prime}, X^{\prime \prime}\right]\right]+\left[X^{\prime},\left[X^{\prime \prime}, X\right]\right]+\left[X^{\prime \prime},\left[X, X^{\prime}\right]\right]
$$

simplifies to,

$$
B \star L_{a b c d} K^{\prime c} K^{\prime \prime d}+B \star L_{a b c d}^{\prime} K^{\prime \prime c} K^{d}+B \star L_{a b c d}^{\prime \prime} K^{c} K^{\prime d}
$$

q.e.d.

Proposition 4. (i) If $V$ is 2-dimensional then any subspace $W$ of $V^{*} \oplus \Lambda^{2} V^{*}$, closed with respect to the bracket, defines a Lie algebra $A(W, B, h)$.

(ii) If $V$ is arbitrary and $B=0$ then $V^{*} \oplus \Lambda^{2} V^{*}$ defines a Lie algebra $A\left(V^{*} \oplus \Lambda^{2} V^{*}, B=0, h\right)$.

\section{Proof:}

(i) Follows from Lemmas 1 and 3. (ii) is immediate. q.e.d.

For local Killing fields $K, K^{\prime} \in K_{U}$, the Lie bracket $K^{\prime \prime}:=\left[K, K^{\prime}\right]$ is,

$$
K_{a}^{\prime \prime}=L_{a b}^{\prime} K^{b}-L_{a b} K^{\prime b}
$$

where we have written $L_{a b}:=K_{a ; b}$ and $L_{a b}^{\prime}:=K_{a ; b}^{\prime}$. Furthermore, $L_{a b}^{\prime \prime}:=K_{a ; b}^{\prime \prime}$ is given by,

$$
L_{a b}^{\prime \prime}=L_{a}^{\prime c} L_{c b}-L_{a c} L_{c b}^{\prime}+R_{a b c d} K^{c} K^{\prime d}
$$

Defining a bracket on $P_{U}$ by,

$$
\begin{aligned}
& {\left[K+L, K^{\prime}+L^{\prime}\right]:} \\
& \quad=L_{a b}^{\prime} K^{b}-L_{a b} K^{\prime b}+L_{a}^{\prime c} L_{c b}-L_{a c} L_{c b}^{\prime}+R_{a b c d} K^{c} K^{\prime d}
\end{aligned}
$$

gives an isomorphism $\phi_{U}: K_{U} \rightarrow P_{U}$ of Lie algebras:

$\phi_{U}\left(\left[K, K^{\prime}\right]\right)=\left[\phi_{U}(K), \phi_{U}\left(K^{\prime}\right)\right]$ 
For $x \in U$, define the subspace $W_{U, x}$ of $W_{x}$ by, $W_{U, x}:=\left\{w \in W_{x}: w=X(x), \quad\right.$ for some $\left.\quad X \in P_{U}\right\}$

Since parallel sections of a vector bundle are determined by their value at a single point, $P_{U}$ and $W_{U, x}$ are isomorphic as vector spaces via the restriction map $r_{x}: P_{U} \rightarrow W_{U, x}$, given by $r_{x}(X):=X(x)$. Comparing (17) and (25), we have, in fact, a Lie algebra isomorphism $r_{x}: P_{U} \rightarrow A\left(W_{U, x}, R_{x}, g_{x}\right)$. Composing this with $\phi_{U}$ characterizes the Lie algebra of $K_{U}$.

\section{Lemma 5.}

$r_{x} \circ \phi_{U}: K_{U} \rightarrow A\left(W_{U, x}, R_{x}, g_{x}\right)$

is an isomorphism of Lie algebras.

In order to find the Lie algebra of the local Killing fields of $g$ about the point $x$ it remains to calculate $W_{U, x}$ for a sufficiently small neighbourhood $U$ of $x$. This is accomplished in the following section.

\section{PARALLEL FIELDS AND LOCALLY SYMMETRIC SPACES}

We begin by briefly reviewing the method from [1]. This describes an algebraic procedure for determining the number of independent local parallel sections of a smooth vector bundle $\pi: W \rightarrow M$ with a connection $\nabla$. Since the existence theory is based upon the Frobenius Theorem, smooth data are required.

Let $W^{\prime}$ be a subset of $W$ satisfying the following two properties:

P1: the fibre of $W^{\prime}$ over each $x \in M$ is a linear subspace of the fibre of $W$ over $x$, and,

P2: $W^{\prime}$ is level in the sense that each element $w \in W^{\prime}$ is contained in the image of a local smooth section of $W^{\prime}$, defined in some neighbourhood of $\pi(w)$ in $M$.

Let $X$ be a local section of $W^{\prime}$. The covariant derivative of $X$ is a local section of $W \otimes T^{*} M$. Define $\widetilde{\alpha}$ by,

$$
\tilde{\alpha}(X):=\phi \circ \nabla(X)
$$

where $\phi: W \otimes T^{*} M \rightarrow\left(W / W^{\prime}\right) \otimes T^{*} M$ denotes the natural projection taken fibrewise. If $f$ is any differentiable function with the same domain as $X$ then,

$$
\tilde{\alpha}(f X)=f \tilde{\alpha}(X)
$$

This means that $\widetilde{\alpha}$ defines a map,

$$
\alpha_{W^{\prime}}: W^{\prime} \rightarrow\left(W / W^{\prime}\right) \otimes T^{*} M
$$

which is linear on each fibre of $W^{\prime}$.

The kernel of $\alpha_{W^{\prime}}$ is a subset of $W^{\prime}$, which satisfies property $\mathrm{P} 1$ but not necessarily property $\mathrm{P} 2$. In order to carry out the above constructions to $\operatorname{ker} \alpha_{W^{\prime}}$, as we did to $W^{\prime}$, the non-level points in $\operatorname{ker} \alpha_{W^{\prime}}$ must be removed. To this end we define a leveling map $S$ as follows. For any subset $V$ of $W$ satisfying P1 let $S(V)$ be the subset of $V$ consisting of all elements $v$ for which there exists a smooth local section $s: U \subseteq M \rightarrow V \subseteq W$ such that $v=s(\pi(v))$. Then $S(V)$ satisfies both $\mathrm{P} 1$ and $\mathrm{P} 2$.

We may now describe the construction of the maximal flat subset $\widetilde{W}$, of $W$. Let,

$$
\begin{aligned}
& V^{(0)} \quad:=\{w \in W \quad \text { I } F(,)(w)=0\} \\
& W^{(i)} \quad:=S\left(V^{(i)}\right) \\
& V^{(i+1)}:=\operatorname{ker} \alpha_{W^{(i)}}
\end{aligned}
$$

where, as before, $F: T M \otimes T M \otimes W \rightarrow W$ is the curvature tensor of $\nabla$. This gives a sequence,

$$
W \supseteq W^{(0)} \supseteq W^{(1)} \supseteq \cdots \supseteq W^{(k)} \supseteq \cdots
$$

of subsets of $W$. For some $k \in N, W^{(l)}=W^{(k)}$ for all $l \geq k$. Define $\widetilde{W}=W^{(k)}$, with projection $\tilde{\pi}: \widetilde{W} \rightarrow M$.

We say that the connection $\nabla$ is regular at $x \in M$ if there exists a neighbourhood $U$ of $x$ such that $\tilde{\pi}^{-1}(U) \subseteq \widetilde{W}$ is a vector bundle over $U . \widetilde{W}_{x}$ shall denote the fibre of $\widetilde{W}$ over $x \in M$.

Lemma 6. Let $\nabla$ be a connection on the smooth vector bundle $\pi: W \rightarrow M$.

(i) If $X: U \subseteq M \rightarrow W$ is a local parallel section then the image of $X$ lies in $\widetilde{W}$.

(ii) Suppose that $\nabla$ is regular at $x \in M$. Then for every $w \in \widetilde{W}_{x}$ there exists a local parallel section $X: U \subseteq M \rightarrow \widetilde{W}$ with $X(x)=w$.

We may now describe the Lie algebra of local Killing fields about a point $x$.

Theorem 7. Let $g$ be a smooth metric on a manifold $M$ with associated connection $\nabla$ on $W=T^{*} M \oplus \Lambda^{2} T^{*} M$, which is assumed to be regular at $x \in M$. Then $g$ has $\operatorname{dim} \widetilde{W}_{x}$ independent local Killing fields in a sufficiently small neighbourhood $U$ of $x \in M$. Moreover, the Lie algebra of Killing fields on $U$ is canonically isomorphic to the Lie algebra $A\left(\widetilde{W}_{x}, R_{x}, g_{x}\right)$.

\section{Proof:}

By Lemma 6 there exists a sufficiently small open neighbourhood $U$ of $x$ such that $W_{U, x}=\widetilde{W}_{x}$. The theorem now follows from Lemma 5 .

q.e.d.

As an illustration, we provide a short algebraic proof of the classical theorem that locally symmetric spaces satisfy,

$R \star R=0$ 
Lemma 8. If $M$ is locally symmetric then $T^{*} M \subseteq \widetilde{W}$.

\section{Proof:}

Suppose $M$ is locally symmetric and let $x \in M$. Then there is an open neighbourhood $U$ of $x$ such that the space of Killing fields on $U$, whose covariant derivative vanishes at $x$, has dimension $n$. By the isomorphism given in Lemma $5, T_{x}^{*} M \subseteq W_{U, x}$. From Lemma 6 (i) we have $W_{U, x} \subseteq \widetilde{W}_{x}$ and so $T_{x}^{*} M \subseteq \widetilde{W}_{x}$.

q.e.d.

Let $T=T_{a \ldots}$ be an $n$-tensor with $n \geq 1$. Define $R \cdot T$ to be the $(n+2)$-tensor obtained by contracting the rightmost index of $R$ with the leftmost index of $T$ :

$R \cdot T_{a b c \cdots}:=R_{a b c}{ }^{s} T_{s \cdots}$

Let $p:=T^{*} M$, the cotangent space of $M$ and let $p^{(1)}$ be defined as the set of all elements $v \in T^{*} M$ satisfying,

$R \star R \cdot v=0$

Define $t$ to be the set of all $L \in \Lambda^{2} T^{*} M$ such that,

$R \star L=0$

We shall assume that $t$ has constant rank.

\section{Proof of (29):}

Let us calculate the derived flag of $W$ supposing $M$ to be locally symmetric. From (8), $W^{(0)}=V^{(0)}:=\operatorname{ker} F=p \oplus t$. Let $X=K+L$ be a local section of $W^{(0)}$, where $K$ and $L$ are local sections of $p$ and $t$, respectively. By definition, $X(x) \in V_{x}^{(1)}$ if and only if $\nabla_{i} X(x) \in W_{x}^{(0)}$, for all $i$. This is equivalent to,

$$
(*): L_{a b ;(i)}-R_{a b(i)}{ }^{c} K_{c} \in t \text { at } x .
$$

Taking the covariant derivative of $R \star L=0$ gives $R \star L_{;(i)}=0$. Thus $L_{a b ;(i)}$ is a local section of $t$. This means that $(*)$ holds if and only if $R \cdot K_{x}$ lies in $t$. This is the case precisely when $K \in p^{(1)}$ at $x$, and so $V^{(1)}=p^{(1)} \oplus t$. By Lemma 8, we must have $V^{(1)}=T^{*} M \oplus t$, whence it follows that $\quad p^{(1)}=T^{*} M \quad$ and $W^{(1)}=V^{(1)}=W^{(0)}$. Therefore $R \star R=0$.

q.e.d.

We have also shown that for $M$ locally symmetric, $\widetilde{W}=W^{(0)}=p \oplus t . \quad$ Conversely, if $\quad \widetilde{W}=p \oplus t \quad$ then $T^{*} M \subseteq \operatorname{ker} F$, from which we conclude that $M$ is locally symmetric. The terminal subbundle of the derived flag therefore characterizes locally symmetric spaces:

$$
M \text { is a locally symmetric space if and only if } \widetilde{W}=p \oplus t \text {. }
$$

In particular, the derived flag computes the local canonical decomposition.
As observed above, a Riemannian manifold with $\operatorname{dim} M \geq 3$ has constant curvature if and only if $R \star L=0$ for all (Proposition 2). Furthermore, all manifolds of dimension $n=1$ or 2 satisfy $R \star L=0$ for $L \in \Lambda^{2} T^{*} M$ (cf. Lemma 1). Since spaces of constant curvature are locally symmetric the curvature $F$ vanishes for such manifolds. It is not difficult to see that the converse holds (for the 2dimensional case use the canonical form of the Riemann curvature: $R_{i j k l}=c\left(g_{i l} g_{j k}-g_{i k} g_{j l}\right)$, where $c$ is the Gaussian curvature). Consequently,

$M$ is a space of constant curvature if and only if $\widetilde{W}=W$.

Employing Theorem 7, we obtain as a corollary the familiar result that a Riemannian manifold possesses the maximal possible number $\frac{1}{2} n(n+1)$ of independent local Killing fields if and only if it is a space of constant curvature.

\section{CLASSIFICATION FOR RIEMANNIAN SURFACES}

In this final section we shall determine which Riemannian surfaces correspond to the various types of Lie algebra $K_{x}$. In the process, a necessary condition for a Riemannian surface to possess a Killing field is obtained. First, we recall the situation involving the maximal number of local Killing fields:

Let $M$ be a Riemannian surface. The following are equivalent:

(i) $\operatorname{dim} K_{x}=3$ for all $x \in M$.

(ii) $M$ has constant Gaussian curvature $c$.

(iii) $M$ is locally symmetric.

In this case,

(a) if $c=0$ then $K_{x}$ is isomorphic to the Lie algebra of $\Re^{2} \times_{s d} S O(2)$;

(b) if $c>0$ then $K_{x} \cong s l_{2} \Re$;

(c) if $c<0$ then $K_{x} \cong s u_{2}$.

The equivalence of (i)-(iii) follows from Lemma 1, (34) and the observation below (34). Assume that these conditions hold. $c=0$ corresponds, locally, to flat Euclidean space, for which $K_{x}$ is isomorphic to the Lie algebra of the semidirect product of translations and rotations. Suppose $c \neq 0$ and let $X$ and $Y$ be orthogonal vectors in $T_{x}^{*} M$ with norm,

$$
X^{2}=Y^{2}=\frac{1}{|c|}
$$

Define $H \in \Lambda^{2} T_{x}^{*} M$ by,

$$
[X, Y]:=H
$$

Then,

$$
[H, X]=-s g(c) Y \quad \text { and } \quad[H, Y]=s g(c) X
$$


where $\operatorname{sg}(c)$ denotes the sign of $c$. Appealing to Lemma 5, this identifies $K_{x}$ with $s l_{2} \Re$ for $c>0$ and with $s u_{2}$ for $c<0$ [5].

Next, we calculate the derived flag for $W$ assuming that the surface is regular: $W^{(i)}=V^{(i)}$ has constant rank for all $i$. The elements $K \in T^{*} M$ satisfying $R_{i j k l}{ }^{; s} K_{s}$ are those for which $c^{s} K_{s}=0$. Therefore, by Lemma 1 ,

$W^{(0)}=\operatorname{ker} \partial c \oplus \Lambda^{2} T^{*} M$

where $\partial c$ denotes the vector field $c^{a}$. The case $\partial c=0$ has been handled above. Suppose therefore that $\operatorname{dim} \operatorname{ker} \partial c=1$; that is, $\partial c$ is non-vanishing. Then $W^{(0)}$ is a rank-two fibre bundle over $M$. Let $X=K+L$ be a local section of $W^{(0)}$, where $K$ is a local section of $\operatorname{ker} \partial c$ and $L$ is a local section of $\Lambda^{2} T^{*} M . \quad X(x) \in W_{x}^{(1)}$ is equivalent to $\nabla_{i} X(x) \in W_{x}^{(0)}$ for all $i$, by the definition of the derived flag. Since $\Lambda^{2} T^{*} M \subseteq W^{(0)}, X(x) \in W_{x}^{(1)}$ if and only if,

$K_{a ;(i)}-L_{a(i)} \in \operatorname{ker} \partial c$

At $x$. Taking the covariant derivative of $c^{, s} K_{s}=0$ gives the equation $c^{, a} K_{a ; b}=-c_{; a b} K^{a}$. Substituting this into (36) determines $W^{(1)}$ as the subset of all $K+L \in W^{(0)}$ satisfying,

$c_{; a b} K^{a}+L_{a b} c^{a}=0$

By contracting (37) with $K$ and $\partial c$, it is evident that $W^{(1)}$ consists of the zero elements in $W$ along with the solutions of,

$c_{; a b} K^{a} K^{b}+L_{a b} c^{a} K^{b}=0$

$c_{; a b} K^{a} c^{, b}=0$

where $0 \neq K \in \operatorname{ker} \partial c$ and $L \in \Lambda^{2} T^{*} M$. Equation (39) has a solution $0 \neq K \in \operatorname{ker} \partial c$ if and only if,

$c_{; a b} c^{, b}=f c_{a}$

for some $f$. (40) may be written in terms of differential forms as,

$d c \wedge D_{\partial c} d c=0$

where $D$ denotes covariant differentiation. This leads to a necessary condition for the existence of a Killing field on a Riemannian surface.

Theorem 9. If a regular Riemannian surface possesses a Killing field then,

$$
d c \wedge D_{\partial c} d c=0
$$

\section{Proof:}

By Theorem 7, if a regular Riemannian surface has a Killing field then $\widetilde{W}$ has rank at least one. Since $\widetilde{W} \subseteq W^{(1)}$, equation (39) must have a non-trivial solution $K \in \operatorname{ker} \partial c$ at each $x \in M$. The theorem now follows from the fact that (39) is equivalent to (41).

q.e.d.

Corollary 10. Let $M$ be a regular Riemannian surface with non-constant curvature. If $M$ possesses a Killing field then the integral curves of $\partial c$ are geodesic paths.

By a geodesic path we mean a curve that is a geodesic when appropriately parameterized.

\section{Proof:}

Equation (41) is equivalent to $D_{\partial c} \partial c=f \partial c$, which implies that integral curves of the non-vanishing vector field $\partial c$ may be parametrized so as to give geodesics of $M$.

q.e.d.

An example would be the punctured paraboloid $z=x^{2}+y^{2} ;(x, y) \neq(0,0)$, with the induced metric from its embedding into 3 -dimensional Euclidean space. The integral curves of $\partial c$ are described by the geodesic paths $\gamma_{\theta}(t)=\left(t \cos \theta, t \sin \theta, t^{2}\right)$, up to reparametrization.

Now let us return to calculating $W^{(1)}$. If $d c \wedge D_{\partial c} d c=0$ on the surface then the non-zero elements of $W^{(1)}$ are the solutions to (38) for which $0 \neq K \in \operatorname{ker} \partial c$. For any choice of non-trivial $K \in \operatorname{ker} \partial c$, (38) uniquely determines an element $L=L(K) \in \Lambda^{2} T^{*} M$. Therefore $W^{(1)}$, in this case, is a rank one vector bundle over $M$. If, on the other hand, $d c \wedge D_{\partial c} d c \neq 0$ on $M$ then $W^{(1)}$ is the zero bundle and there do not exist any local Killing fields. As a consequence, $K_{x}$ cannot be 2-dimensional; this may also be seen directly by considering the Lie bracket operation. Henceforth we shall assume that $d c \wedge D_{\partial c} d c=0$.

To find $W^{(2)}$, let $X=K+L$ be a local section of $W^{(1)}$. By definition, $X(x) \in W_{x}^{(2)}$ if and only if $\nabla_{i} X(x) \in W_{x}^{(1)}$ for all $i$. Owing to (37), this is equivalent to

$c_{; a b} K_{(i)}^{\prime a}+L_{a b(i)}^{\prime} c^{, a}=0$

Where,

$$
\begin{gathered}
K_{a(i)}^{\prime}:=K_{a ;(i)}-L_{a(i)} \\
L_{a b(i)}^{\prime}:=L_{a b ;(i)}-R_{a b(i)}{ }^{c} K_{c}
\end{gathered}
$$

(Note that from the description of $W^{(1)}$ contained in (36) it follows that $\left.K_{a(i)}^{\prime}+L_{a b(i)}^{\prime} \in W^{(0)}\right)$. Taking the covariant derivative of (37) gives,

$c_{; a b} K_{;(i)}^{a}+L_{a b ;(i)} c^{a}=-c_{; a b(i)} K^{a}-L_{a b} c_{(i)}^{; a}$

Substituting (43) into (42) defines $W^{(2)}$ as the subset of all $K+L \in W^{(1)}$ such that,

$c_{; a b c} K^{a}+L_{a b} c^{; a}{ }_{c}+L_{a c} c^{; a}{ }_{b}+R_{a b c d} c^{, a} K^{d}=0$ 
If this has only the trivial solution then $\widetilde{W}=W^{(2)}$ is the zero bundle. Otherwise, $\widetilde{W}=W^{(2)}=W^{(1)}$ has rank one.

Theorem 11. Let $M$ be a regular Riemannian surface. Then $\operatorname{dim} K_{x}=1$ for all $x \in M$ if and only if,

(i) $d c \neq 0$,

(ii) $d c \wedge D_{\partial c} d c=0$, and

(iii) equation (44) holds for all $K+L \in W^{(1)}$.

\section{Proof:}

Conditions (i)-(iii) are equivalent to $\operatorname{rank} \widetilde{W}=1$. The result now follows from Theorem 7.

q.e.d.

We summarize the discussion in this section with the following corollary.

Corollary 12. For regular Riemannian surfaces, $K_{x}$ may be one of five possible Lie algebras. It is isomorphic to either $s l_{2} \mathfrak{R}, \quad s u_{2}$ or the Lie algebra of $\Re^{2} \times_{s d} S O(2)$ when the Gaussian curvature is constant and positive, negative or zero, respectively. $K_{x}$ is the 1-dimensional Lie algebra when the conditions of Theorem 11 are met. Otherwise, there do not exist any local Killing fields and $K_{x}$ is trivial.

\section{REFERENCES}

[1] Atkins R. Existence of Parallel Sections of a Vector Bundle. JGP 2011; 61: 309-11.

[2] Kostant B. Holonomy and the Lie algebra of infinitesimal motions of a Riemannian manifold. Trans Amer Math Soc 1955; 80.

[3] Console S, Olmos C. Curvature invariants, Killing vector fields, connections and cohomogeneity. Proc Am Math Soc 2009; 137(3): 1069-72.

[4] Helgason S. Differential geometry, lie groups, and symmetric spaces. Graduate studies in mathematics American Mathematical Society 2001.

[5] Fulton W, Harris J. Representation theory. Graduate texts in mathematics. Springer 1991

Received: November 04, 2009

Revised: December 28, 2010

Accepted: February 28, 2011

(C) Richard Atkins; Licensee Bentham Open.

This is an open access article licensed under the terms of the Creative Commons Attribution Non-Commercial License (http://creativecommons.org/licenses/by$\mathrm{nc} / 3.0 /$ ), which permits unrestricted, non-commercial use, distribution and reproduction in any medium, provided the work is properly cited. 